\title{
Electron Holography and Micromagnetic Simulations for TEM Magnetization Mapping
}

\author{
M. Beleggia ${ }^{*}$, M.A. Schofield, Y. Zhu*, M. Malac ${ }^{*, * *}$, Z. Liu ${ }^{* *}$ and M. Freeman ${ }^{* *}$ \\ *Department of Materials Science, Brookhaven National Laboratory, Upton, Long Island, NY 11973 \\ ${ }^{* *}$ Department of Physics, University of Alberta, Edmonton T6G 2J1, Canada
}

A very special care is needed to retrieve quantitatively the magnetic configuration of nanoparticles in TEM. The two main issues still to be properly addressed before reaching a truly quantitative level are the separation of the electrostatic and magnetic contributions to the phase shift, and the fringing field contribution to the magnetic signal. Nanoparticles are almost never flat, and often resemble a spherical geometry. Therefore we cannot assume that the electrostatic contribution to the phase shift is a constant, which can be neglected at least far from the particle edges. Moreover, the absence of fringing field is granted only in an ideal closure-domain magnetic state, where no magnetic field is leaking outside the particle. In a real particle, fringing fields are always present, and the magnetic signal is actually the projection of the magnetic induction averaged along the electron trajectory. It has been shown [1] that neglecting the fringing field can be misleading in the image interpretation. A uniformly magnetized particle appears in projection as a non-uniform magnetic structure. Hence, if the fringing fields are not correctly taken into account, the projected magnetic field distribution retrieved from the experiment cannot be directly related to the magnetization of the particle.

In this work we address the problem of fringing fields arising from a permalloy element. Thin film samples of permalloy squares and rectangles on $50 \mathrm{~nm}$ thick silicon nitride membrane were prepared by electron beam patterning of PMMA photoresist followed by lift off of a sputter deposited film. The permalloy film was deposited in a field-free environment. To prevent charging in the electron beam the sample was coated with a $2 \mathrm{~nm}$ thick, amorphous carbon layer. The composition of the permalloy film was measured to be $\mathrm{Ni} 88 \%$ Fe $12 \%$ by electron energy loss spectroscopy using a JEOL $3000 \mathrm{~F}$ TEM. The size of the element is approximately $830 \times 855 \times 45 \mathrm{~nm}^{3}$. To better match the element shape, the in-focus TEM image was employed as a template to define the geometry in the micromagnetic simulation.

By an extension of our Fourier-space approach for the calculation of the electron optical phase shift $[2,3]$, it is now possible to connect the output of a micromagnetic simulation on a nanoparticle, which usually shows a complex magnetic configuration, with the effect of the particle itself on the electron beam, namely the total magnetic phase shift. A micromagnetic simulation, based on Landau-Lifshitz-Gilbert equation, shows the evolution of the magnetic configuration from an initial random state to a final vortex state (Fig. 1). The corresponding magnetic phase shift for the final four states is displayed in Fig. 2, where it can be seen in the right column that fringing fields around the particle are present and contribute significantly to the phase shift. The excellent agreement between the phase shift retrieved from an electron holography experiment (Fig. 3) and the obtained phase shift for the final vortex state, Fig. 2(d), allows us to claim that the magnetic configuration of the final state of Fig. 1 is the real, quantitative, map of the element magnetization.

It has been shown that a truly quantitative magnetization mapping of sub-micron magnetic elements by TEM is possible. The magnetic configuration of a permalloy $\mathrm{Ni}_{88} \mathrm{Fe}_{12}$ element has been obtained 
by the combination of electron holography, micromagnetic simulations and phase shift calculations. Experiments are now in progress to investigate magnetic elements and nanoparticles at a scale down to a few nanometers in size [4].

\section{References}

[1] M. Beleggia et al., Philos. Mag. B 83 (2003) 1143.

[2] M. Beleggia and Y. Zhu, Philos. Mag. B 83 (2003) 1043.

[3] M. Beleggia et al., Ultramicroscopy, in press (2003).

[4] This work was supported by U.S. Department of Energy, Basic Energy Science, under contract No. DE-AC02-98CH10886

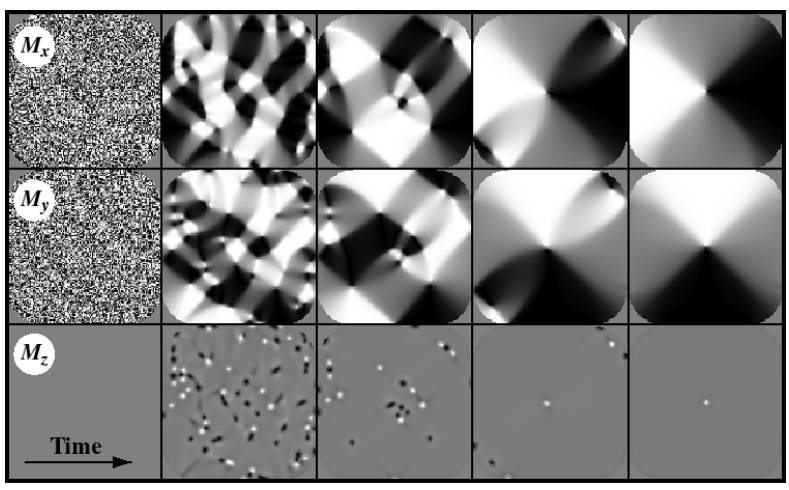

Fig.1: Micromagnetic simulation on the permalloy element. An initial random state (left) evolves to a vortex state (right).
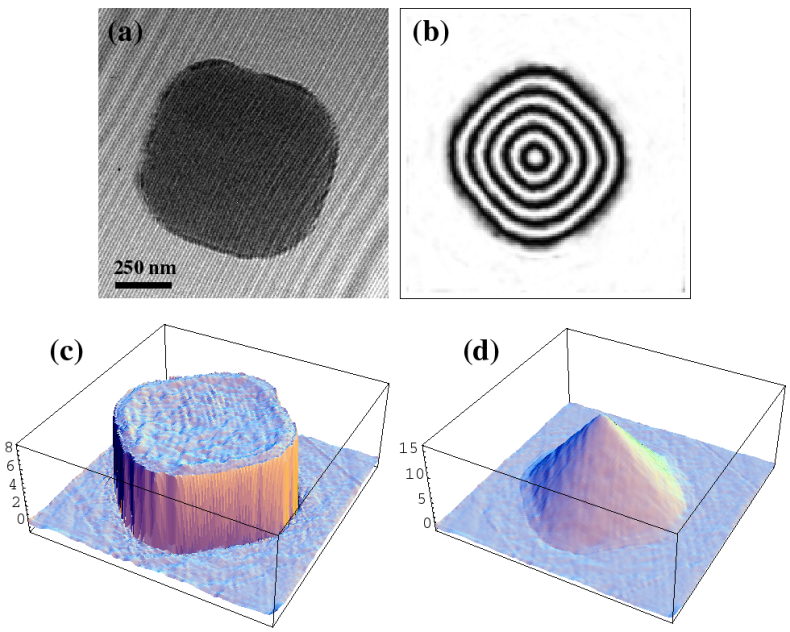

Fig. 3: \{(a) Electron hologram of the magnetic element; (b) Contour map (2x amplified) of the total phase shift; (c) Electrostatic and (d) Magnetic contributions to the phase shift (in radiants) retrieved from the experiment.

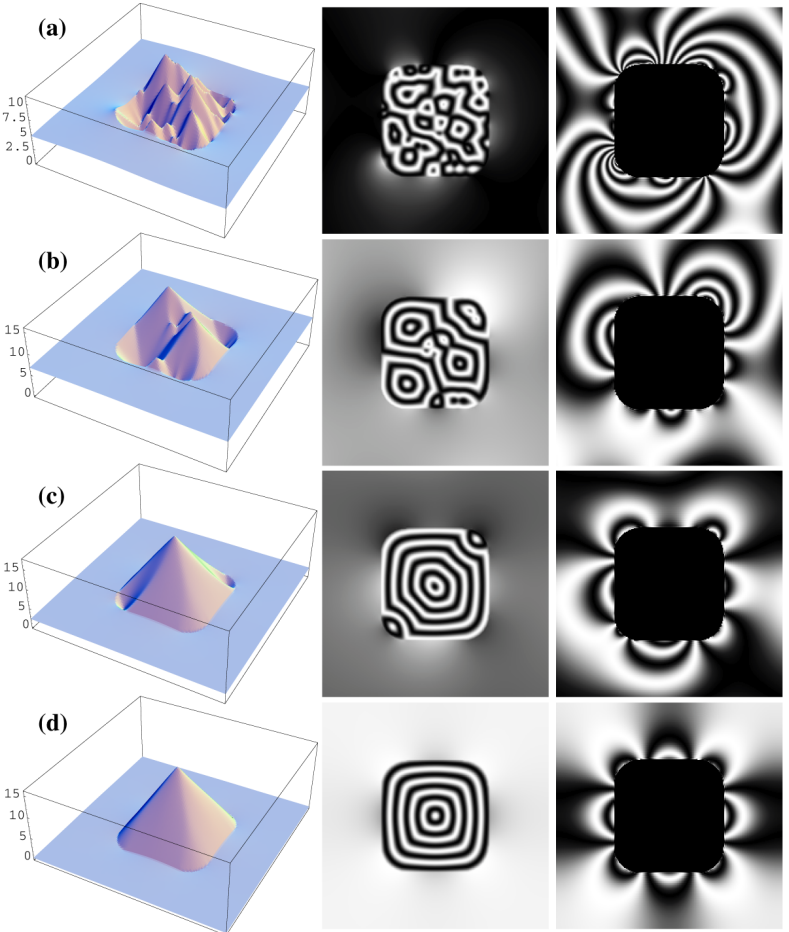

Fig.2: Phase shift from micromagnetic simulations (last four states of Fig. 1) displayed as 3D plot (first column), 2x (second column) and $32 x$ (third column) amplified contour maps. 